\title{
Textile Science Behind the Mask Homemade with Love Filtered Cloth Mask
}

\author{
Sally M Di Marco*
}

Stevenson University, USA

*Corresponding author: Sally M Di Marco, Associate Professor and Fashion Design Program Coordinator (Retired), Department of Fashion Design, Stevenson University, USA.

Received Date: January 28, 2021

Published Date: February 05, 2021

\section{Abstract}

In order to address the needs of the current Covid-19 Pandemic for a safe face mask that can be worn for everyday wear, published scientific research was culled and utilized in the design and materials for creating the Homemade with Love (HWL) filtered cloth mask [1] (Figure 1). Researchers from the Argonne National Laboratory at the University of Chicago in the United States reported that high thread counts 100 percent plain woven cotton or cotton blends, along with a combination of layering of different fabrics, with electrostatic-based filtration produces a mask that can block "a vast majority of aerosol particles." The HWL facemask meets the researcher's findings, and it is compliant with the Center for Disease Control (CDC) guidelines for homemade masks [2].

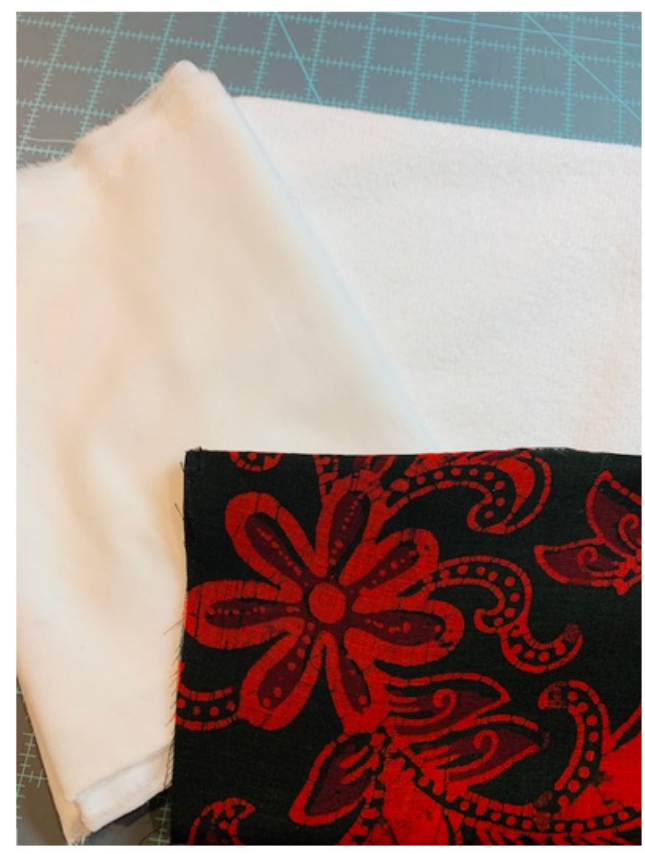

Figure 1: Materials for the HWL mask: cotton lining (100\% cotton), electrostatic filter ( $80 \%$ polyester and $20 \%$ Polyamide), fashion fabric is a printed Batik from Africa backed with quilters cotton ( both $100 \%$ cotton). 


\section{Anatomy of the Mask}

\section{Style and fit}

Mirrored after the surgical mask, the pleated design of the HWL mask contributes to the fit, skin comfort, and most importantly allows for adequate amount of airflow. According to the CDC article (2020) entitled, Considerations for Wearing Masks, "wearing a mask does not raise the carbon dioxide $\left(\mathrm{CO}_{2}\right)$ level in the air you breathe. A cloth mask does not provide an airtight fit across the face. The $\mathrm{CO}_{2}$ completely escapes into the air through and around the sides of the cloth mask when you breathe out or talk. $\mathrm{CO}_{2}$ is small enough to easily pass through any cloth mask material. In contrast, the virus that causes COVID-19 is much larger than $\mathrm{CO}_{2}$, so it cannot pass as easily through a properly designed and properly worn cloth mask." Additionally, the style of the mask minimizes the build-up of condensation, which can be a source of bacterial growth. The mask molds across the face, is gentle to the skin, and protective. Because it utilizes three layers of fabrics, it can be considered as a form of quilting, creating more padding in the construction process, and protection when worn (Figure 2).

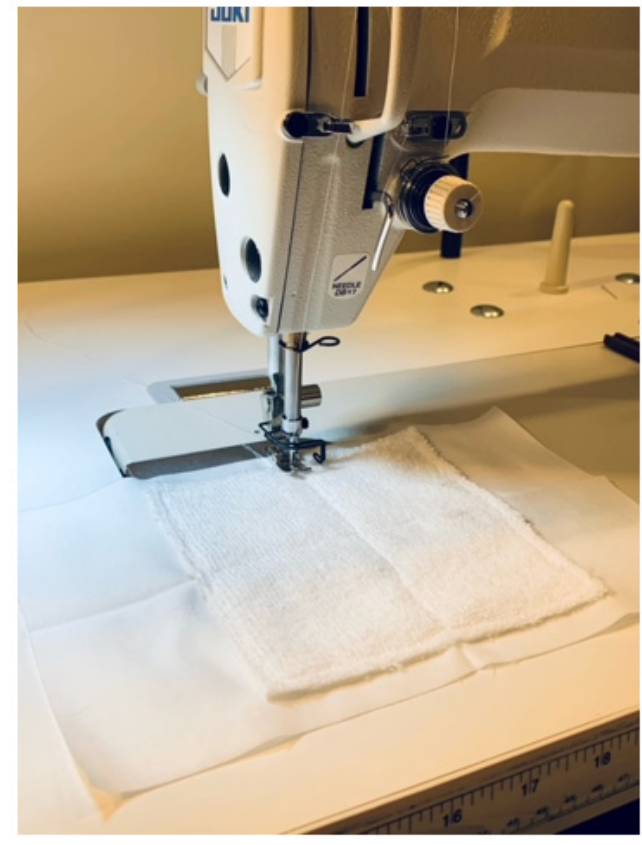

Figure 2: Mask making process: Attaching the electrostatic filter to the lining of the mask.

Cutting the mask on the crosswise grain of the fabric, where the give of the fabric is predominant, allows for the mask to slightly stretch and accommodate the contours of the face. The crosswise or weft yarns run perpendicular to the selvage of the fabric. The selvage is the finished edge, on both sides of the fabric, which runs lengthwise and serves to finish off the edges. Adding purchased adjustable earpieces and a nose bridge further contributes to a well-fitting mask that will not slip and covers both the nose and the mouth.

\section{Textile Science Behind the Mask}

\section{Public face of the mask}

The HWL filtered mask is made exclusively out of quilter's cotton for the fashion fabric (public side of the mask) and the lining (private side of the mask). Quilters 100 percent cotton is a plain, tightly woven, medium weight textile. It is crisper than standard cotton, absorbent, dries quickly, and holds up in the laundering process. The fabric is available in solid colors or printed after it is woven in a variety of patterns. Due to the nature of the fabric, it will withstand numerous washing cycles in cold and hot temperatures (Figure 3).

Faced with the possibility that there could be a shortage of masks, Wake Forest Baptist Medical Center tested 13 different designs and an estimated 400 homemade cloth surgical masks. Scott Segal, M.D., Chair of Anesthesiology conceived the idea. "What the test team found was that the masks' effectiveness varied widely. The best homemade masks achieved 79\% filtration as compared to surgical masks (62\% to 65\%) and N95 masks (97\%). But other homemade masks tested performed significantly worse, sometimes demonstrating as little as $1 \%$ filtration. The best performing design was constructed of two layers of high-quality, heavyweight

"quilter's cotton" with a thread count of 180 or more, and those with especially tight weave and thicker thread such as batiks. A double-layer mask with a simple cotton outer layer and an inner layer of flannel also performed well, Dr. Segal said." The testing was conducted at the Manufacturing Development Center at the Wake Forest Institute for Regenerative Medicine [3]. 


\section{Private face of the mask: Lining}

The HWL filtered mask is lined with 100 percent quilter's cotton or a high-grade muslin in white, cream, or black. It is not advisable to line the mask in the fashion fabric if it is printed. To have a heavily dyed print fabric next to the face, especially near the mouth and nose is problematic and may cause allergic reactions in susceptible individuals (Figure 4).

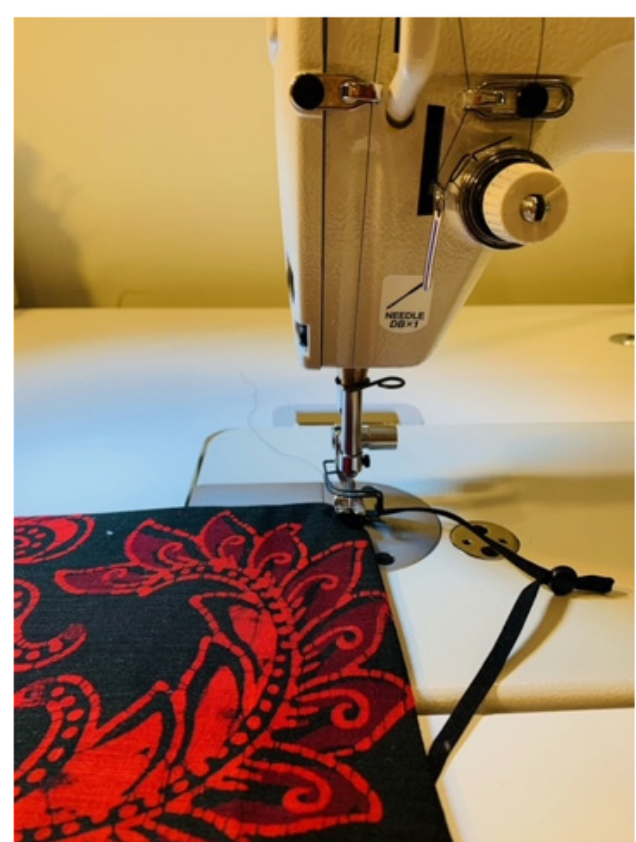

Figure 3: Mask making process: Sewing the fashion fabric and the earpieces to lining of the mask. The nose bridge has been sandwiched between the lining and the fashion fabric at the top of the mask (not shown in the photo).

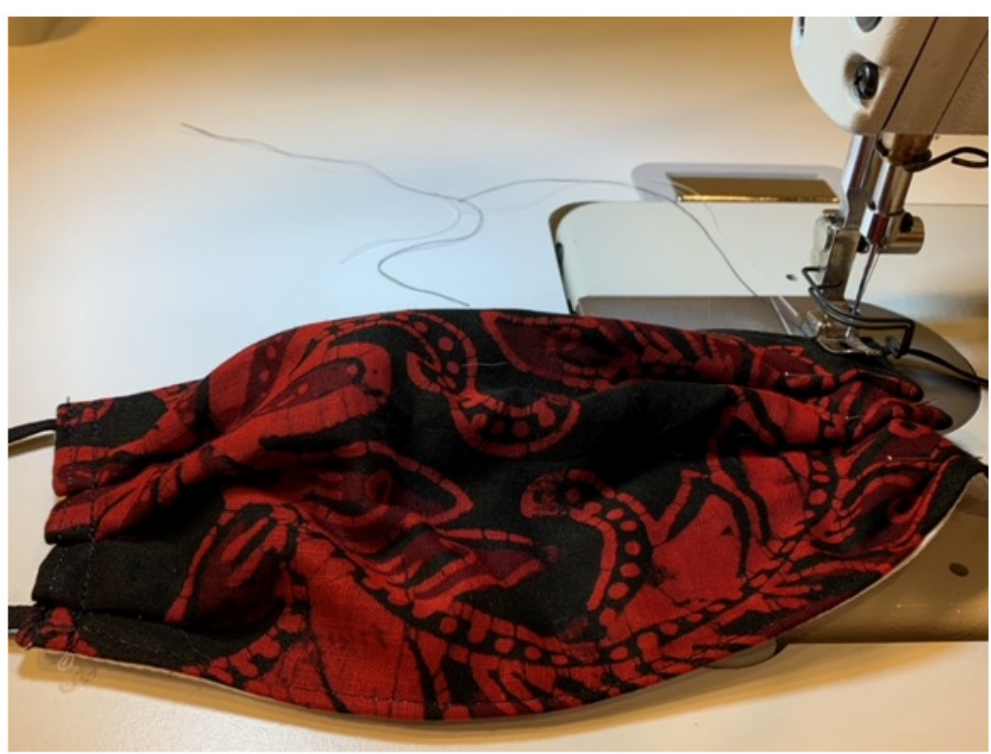

Figure 4: Mask Making Process: Stitching the pleats on each side of the mask.

\section{Electrostatic filter}

In order to attempt to block aerosol particles, it's recommended that an electrostatic filter be built into the mask. The HWL includes a permanent microfiber towel fabric as the filtering agent. Microfiber fabrics are positively charged and attract and trap particles by generating static electricity that acts like a magnet.
One of the most effective products on the market are multipurpose microfiber washing and drying towels made out of 80 or 90 percent polyester and 10 or 20 percent polyamide (nylon). The 80 percent to 20 percent ratio is the most popular and effective blend. The high absorbency and quick drying aspect of this fabric is critical in skin comfort and keeping bacteria count at bay. It is important to note 
when using this fabric that bleach or fabric softener cannot be used in the washing cycle because it will neutralize the positive charge of the cloth.

Studies conducted by researchers at the Argonne National Laboratory in Chicago also supported using electrostatic-based filtration such as the microfiber towels as the filtering medium. According to researchers, "electrostatic-based filtration is a little different. Think of a super static-y material such as polyester. Instead of zapping a friend with all the static electricity you saved up, the electrostatic filter keeps the aerosols inside the static environment" (Figures 5\&6).

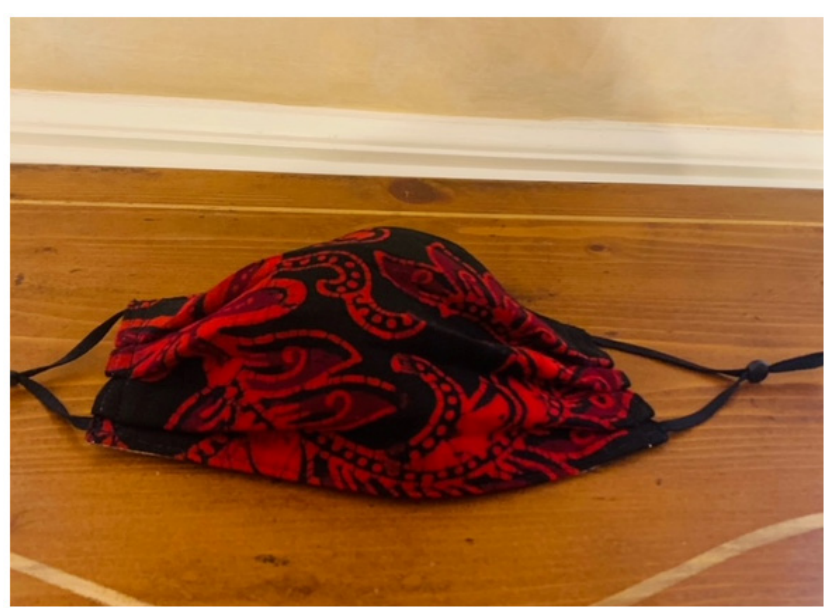

Figure 5: HWL Mask: The finished protective mask.

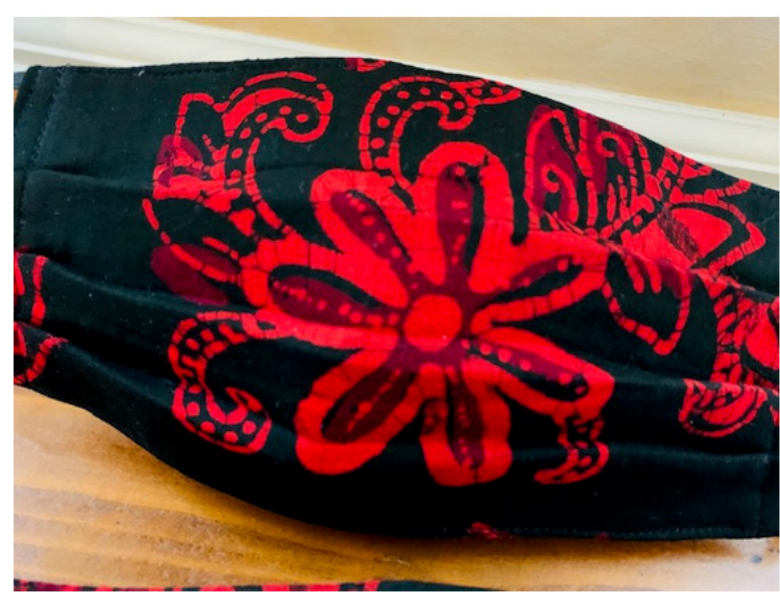

Figure 6: HWL Mask: The finished protective mask.

\section{Conclusion}

A protective homemade facemask can be constructed using a combination of high thread count 100 percent quilters cotton, a built-in microfiber electrostatic filter, and a combination of layered materials such as cotton and a polyester and nylon filter. Regardless, homemade masks should not be used in medical settings or as a

replacement for the N95 medical masks. Homemade masks are to be worn by the general population for daily life only.

\section{Acknowledgement}

Francesca Di Marco, Digital Content Developer, HWL website developer and manager; Dr. Frank Monteferrante, Environmental
Scientist, HWL proofreading and editing; Erika Yuille, Fashion Designer, HWL product development.

\section{Conflict of Interest}

Author declare no conflict of interest.

\section{References}

1. (2020) Scientists have figured out the best materials to use if you're making a mask at home.

2. (2020) Considerations for wearing masks, center for disease control (CDC).

3. (2020) Testing shows type of cloth used in homemade masks makes a difference, doctors say. 